\section{Constraining the time evolution of dark energy, curvature and neutrino properties with cosmic chronometers}

To cite this article: Michele Moresco et al JCAP12(2016)039

View the article online for updates and enhancements.

\section{Related content}

- New constraints on cosmological
parameters and neutrino properties using
the expansion rate of the Universe to z
Michele Moresco, Licia Verde, Lucia
Pozzetti et al.
- Early cosmology constrained
Licia Verde, Emilio Bellini, Cassio Pigozzo
et al.
- Ultra-compact structure in radio quasars
as a cosmological probe: a revised study
of the interaction between cosmic dark
Sectors
Xiaogang Zheng, Marek Biesiada, Shuo
Cao et al.

\section{Recent citations}

$$
\begin{aligned}
& \text { A SHARP view of HOLiCOW: HO from } \\
& \text { three time-delay gravitational lens systems } \\
& \text { with adaptive optics imaging } \\
& \text { Geoff C-F Chen et al } \\
& \text { - Baryon acoustic oscillation, Hubble } \\
& \text { parameter, and angular size measurement } \\
& \text { constraints on the Hubble constant, dark } \\
& \text { energy dynamics, and spatial curvature } \\
& \text { Joseph Ryan et al } \\
& \text { - Exploring neutrino mass and mass } \\
& \text { hierarchy in the scenario of vacuum } \\
& \text { energy interacting with cold dark matter } \\
& \text { Rui-Yun Guo et al }
\end{aligned}
$$

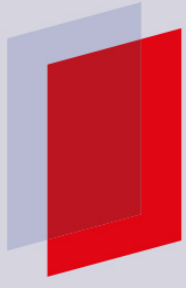

\section{IOP ebooks'}

Bringing you innovative digital publishing with leading voices to create your essential collection of books in STEM research. Start exploring the collection - download the first chapter of every title for free. 


\section{Constraining the time evolution of dark energy, curvature and neutrino properties with cosmic chronometers}

\section{Michele Moresco, ${ }^{a, b}$ Raul Jimenez, ${ }^{c, d, e}$ Licia Verde, ${ }^{c, d, e, f}$ Andrea Cimatti, ${ }^{a}$ Lucia Pozzetti, ${ }^{b}$ Claudia Maraston ${ }^{g}$ and Daniel Thomas $^{g}$}

\footnotetext{
${ }^{a}$ ALMA Mater Studiorum - Università degli Studi di Bologna, Dipartimento di Astronomia, via Ranzani 1, Bologna, I-40127 Italy

${ }^{b}$ INAF - Osservatorio Astronomico di Bologna, via Ranzani 1, Bologna, 40127 Italy

${ }^{c}$ ICREA, Pg. Lluis Companys 23, Barcelona, 08010 Spain ${ }^{d}$ ICC, University of Barcelona (UB-IEEC), Marti i Franques 1, Barcelona, 08028 Spain

${ }^{e}$ Radcliffe Institute for Advanced Study, Harvard University, 8 Garden Street, Cambridge, MA, 02138 U.S.A.

${ }^{f}$ Institute of Theoretical Astrophysics, University of Oslo, Sem Sælands vei 13, Oslo, 0371 Norway

${ }^{g}$ Institute of Cosmology and Gravitation, Dennis Sciama Building, University of Portsmouth, Burnaby Road, Portsmouth, PO1 3FX U.K.

E-mail: michele.moresco@unibo.it,raul.jimenez@icc.ub.edu, liciaverde@icc.ub.edu, a.cimatti@unibo.it, lucia.pozzetti@oabo.inaf.it, claudia.maraston@port.ac.uk, daniel.thomas@port.ac.uk
}

Received April 4, 2016

Revised November 1, 2016

Accepted December 1, 2016

Published December 22, 2016 
Abstract. We use the latest compilation of observational Hubble parameter measurements estimated with the differential evolution of cosmic chronometers, in the redshift range $0<$ $z<2$, to place constraints on cosmological parameters. We used a Markov-Chain MonteCarlo approach to sample the parameter space for the cosmic chronometers dataset alone and in combination with other state-of-the art cosmological measurements: CMB data from the latest Planck 2015 release, the most recent estimate of the Hubble constant $H_{0}$, a compilation of recent baryon acoustic oscillation data, and the latest type Ia cosmological supernovae sample. From late-Universe probes alone $(z<2)$ we find that $w_{0}=-0.9 \pm 0.18$ and $w_{a}=$ $-0.5 \pm 1.7$, and when combining also Planck 2015 data we obtain $w_{0}=-0.98 \pm 0.11$ and $w_{a}=-0.30 \pm 0.4$. These new constraints imply that nearly all quintessence models are disfavoured by the data; only phantom models or a pure cosmological constant are favoured. This is a remarkable finding as it imposes severe constraints on the nature of dark energy. For the curvature our constraints are $\Omega_{k}=0.003 \pm 0.003$, considering also CMB data. We also find that $H(z)$ data from cosmic chronometers are important to constrain parameters that do no affect directly the expansion history, by breaking or reducing degeneracies with other parameters. We find that $N_{\text {eff }}=3.17 \pm 0.15$, thus excluding the possibility of an extra (sterile) neutrino at more than $5 \sigma$, and put competitive limits on the sum of neutrino masses, $\Sigma m_{\nu}<0.27 \mathrm{eV}$ at $95 \%$ confidence level. Finally, we constrain the redshift evolution of dark energy by exploring separately the early and late-Universe, and find a dark energy equation of state evolution $w(z)$ consistent with that in the $\Lambda$ CDM model at the \pm 0.4 level over the entire redshift range $0<z<2$.

Keywords: dark energy experiments, dark energy theory, neutrino masses from cosmology

ArXiv ePrint: 1604.00183 


\section{Contents}

1 Introduction 1

2 Data 2

2.1 Cosmic chronometers dataset 3

2.2 Additional datasets 3

3 Methodology 4

4 Results $\quad 7$

4.1 Constraints from the late-Universe probes $\quad 7$

4.2 Combining early-Universe information 11

5 Conclusions

A Dependence of the results on the assumed evolutionary stellar population synthesis model

\section{Introduction}

Currently, the $\Lambda \mathrm{CDM}$ model represents the simplest framework to describe all available cosmological information. Within this model, the Universe has no spatial curvature, the present day energy is mostly constituted of a dark energy component in the form of a cosmological constant, and there are three massless neutrinos. This model can accurately match at the $\sim$ percent level current observations with the minimal number of parameters.

One approach to make progress in understanding the nature of dark energy is to measure quantities that are independent of the cosmological model. One such technique is to measure directly the expansion history of the Universe: this can be done using massive and passively evolving early-type galaxies as "cosmic chronometers" [1], thus providing standard(-izable) clocks in the Universe. The basic idea underlying this approach is based on the measurement of the differential age evolution as a function of redshift of these chronometers, which provides a direct estimate of the Hubble parameter $H(z)=-1 /(1+z) d z / d t \simeq-1 /(1+z) \Delta z / \Delta t$. The properties of these tracers ensure that they are the best candidates to exploit this technique: they are extremely homogeneous in terms of redshift of formation, assembled their mass at high redshifts $(z \sim 2-3)$, are passively evolving since then, hence representing the oldest objects in the Universe at each redshift. An extensive discussion of the method is provided in refs. $[2,3]$. There has been significant progress both in the modeling and control of systematics uncertainties $[4,5]$, and in the improvement of observational data $[2,3,6]$; in particular in these latter works the method was exploited providing new accurate measurements of $H(z)$ in the redshift range $0<z<2$, that were used alone [6] and in combination with other probes [5] to constrain cosmological parameters. It is important to underline that the main strength of this approach is that it measures a differential quantity, $\Delta z / \Delta t$, which minimizes many issues and systematic effects (for a detailed discussion, see [2]).

The cosmic chronometer approach relies on the determination of accurate stellar ages for galaxies. These are determined by fitting stellar population models to data, either photometric or spectroscopic. Hence the quality of the derived ages depends on the following 
entries and their interplay: the input stellar population model, which in turn depends on the input stellar models; the fitting procedure, e.g. principal component, reconstructed star formation history, parametric star formation history; the wavelength range encompassed by the data. Several studies have recently assessed the impact of various effects on the robustness of ages (e.g. [7, 8]). These works illustrate that spectral fitting is now a robust procedure. Ultimately, the internal clock is dictated by the equation of state assumed in the stellar models and the nuclear reaction rates. For example, ref. [9] finds that using models based on so-called Padova tracks or Frascati tracks lead to a shift of $500 \mathrm{Myr}$ in age for populations about 1 Gyr old, and smaller for older populations.

Because the cosmic chronometer method is independent of the assumed cosmological model, it can provide constraints on the parameters that govern the expansion history of the Universe in a way that can be used to test cosmological models. The most interesting parameters to be constrained are those affecting the background evolution, chiefly the evolution of dark energy and the curvature. However, other parameters which from Cosmic Microwave Background data alone show degeneracies with the background evolution, such as neutrino properties, can also be constrained.

The main aim of this paper is to explore what constraints, on models beyond $\Lambda$ CDM, can be obtained from the the new dataset of $H(z)$ measurements from ref. [3] both alone and in combination with other data. In particular, we focus on constraints on the time evolution of dark energy, and we demonstrate how early and late-Universe probes give a consistent picture. Further, we demonstrate that the new $H(z)$ data, in combination with the other late-Universe probes, allow for a reconstruction with up to 3 free parameters of the time evolution of dark energy, when dark energy is parameterised via a Chebyshev expansion. We also explore the standard Chevallier-Polarski-Linder (CPL) parameterization, providing accurate constraints on dark energy evolution. The paper is organised as follows. In section 2 we present the data, both the $H(z)$ data and the other state-of-the-art cosmological data and in section 3 we state our underlying assumptions and the methodological approach. In section 4 we present our results, first for the cosmic chronometers data alone, then in comparison with external data sets and finally in combination, discussing our constraints on dark energy time evolution, curvature, and neutrino properties. We conclude in section 5.

\section{Data}

In this analysis, we compare the constraints on cosmological parameters that can be obtained by probes that map the late-time Universe $(z<2)$ expansion history, as well as the improvement that can be obtained by combining those with early-time Universe probes. The baseline of our analysis is the Hubble parameter measurements obtained with the cosmic chronometers (hereafter CC) technique, but we consider as well more "standard" probes such as Supernovae Type Ia (hereafter SNe), Baryon Acoustic Oscillation distance measurements (hereafter $\mathrm{BAO}$ ), and local $H_{0}$ measurements (hereafter $H_{0}$ ). Early-time Universe probes are the latest Cosmic Microwave Background (CMB) measurements from the Planck mission (hereafter Planck15). We use directly the posterior sampling provided by the Planck collaboration for specific models and for the full combination of temperature and polarisation power spectrum data (in the Planck15 nomenclature TT,TE,EE+lowP). ${ }^{1}$ We refer to ref. [10] for more information. In the following we present the other datasets considered.

\footnotetext{
${ }^{1}$ Downloadable from http://pla.esac.esa.int/pla/\#cosmology under "Full grid of results".
} 


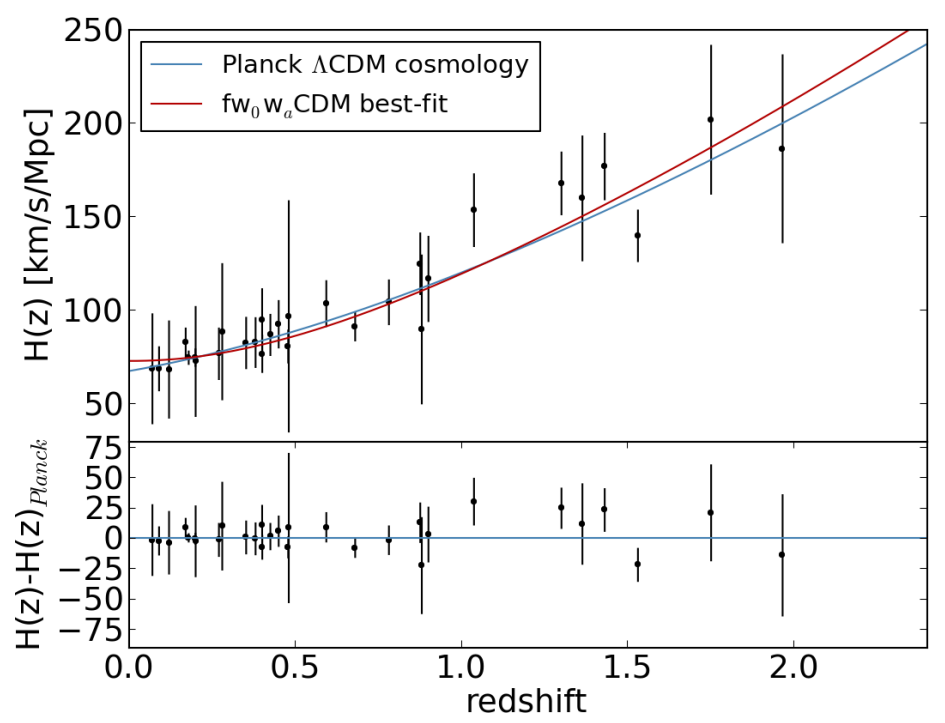

Figure 1. Cosmic chronometers dataset used in this analysis. The red line show the best-fit to the data assuming a $\mathrm{fw}_{0} \mathrm{w}_{a}$ CDM cosmology, while the blue line show the fiducial Planck $\Lambda$ CDM cosmology $\left(H_{0}=67.8 \mathrm{~km} / \mathrm{s} / \mathrm{Mpc}, \Omega_{m}=0.308\right)$. Lower panels show the residuals of the data with respect to the fiducial Planck cosmology. CC data have been taken from refs. [2, 3, 6, 11-13].

\subsection{Cosmic chronometers dataset}

The cosmic chronometers approach to measure $H(z)$ was first introduced in ref. [1]; it uses relative ages of the most massive and passively evolving galaxies to measure $d z / d t$, from which $H(z)$ is inferred. The latest implementation has been explained in detail in ref. [2], where the possible sources of uncertainty and related issues are also discussed; we refer to those references for a comprehensive discussion. We consider the compilation of Hubble parameter measurements provided by [3]. It contains the latest updated list of $30 H(z)$ measurements [2, $3,6,11-13]$ spanning the redshift range $0<z<2$. This sample covers roughly 10 Gyr of cosmic time; the data are presented in figure 1 . The $\mathrm{CC}$ approach to measure $H(z)$ has the desirable feature of being largely independent on assumptions about the cosmological model (besides isotropy and homogeneity); it does, however, rely on the identification of an optimal tracer of the aging of the Universe with redshift (a cosmic chronometer), and a reliable way to measure the age of a tracer ("dating"). An extended discussion can be found in ref. [3]. Of particular importance is a possible dependence on the adopted evolutionary stellar population synthesis (EPS) model, which is key in determining the age of the chronometer. In this analysis we used the measurements calibrated on [18] (BC03) EPS models, since they provide the largest dataset to date. However, refs. $[2,3,6]$ provided measurements also with the newest M11 [19] models for a smaller dataset. We explore the dependence of our results on the adopted EPS model in appendix A.

\section{$2.2 \quad$ Additional datasets}

More standard cosmological probes have been also exploited as complementary datasets to this analysis.

Type 1A supernovae (standard candles). We consider the latest "joint light curves" (JLA) sample [14], comprising 740 SNe Ia from the three year Sloan Digital Sky Survey, 
Supernova Legacy Survey [20, 21], Hubble Space Telescope [22, 23] and other local experiments (see [24]). Here we use the binned distance modulus provided by ref. [14], with its associated covariance matrix. It is defined as:

$$
\mu_{b}=M+5 \log _{10} D_{L}(z),
$$

where $\mathrm{M}$ is a (nuisance) normalization parameter and $D_{L}(z)$ the luminosity distance at redshift $z$. The luminosity distance at redshift $z$ is related to an integral of the Hubble parameter from redshift 0 to $z$. As such, it offers sensitivity to the curvature parameter but its integral nature makes it less sensitive than CC to sharp variations in $H(z)$. Also marginalisation over $M$ makes this probe sensitive to the shape of $H(z)$ but not to its overall normalisation (characterised for example by the value at a given redshift like $\left.z=0, H_{0}\right)$.

Baryon Acoustic oscillation (standard rulers). Our BAO analysis is based on the (isotropic) acoustic-scale distance ratio $\mathrm{D}_{\mathrm{V}}(\mathrm{z}) / \mathrm{r}_{\mathrm{drag}}$ where $r_{\mathrm{drag}}$ is the sound horizon at radiation drag,

$$
D_{V}(z)=\left[(1+z)^{2} D_{\mathrm{A}}^{2}(z) \frac{c z}{H(z)}\right]^{1 / 3}
$$

and $D_{\mathrm{A}}(z)$ is the angular diameter distance at redshift $z$.

Our compilation comprises the measurements obtained by 6dFGS [15], SDSS Main Galaxy Sample [16] and BOSS LOWZ and CMASS surveys [17] and is similar to the baseline BAO dataset used in Planck 2015 analysis [10]. $D_{V}$ is a combination of an integral of $H(z)$ (through $D_{\mathrm{A}}$ ) and a direct $H(z)$ measurement, ${ }^{2}$ but the exquisite measurement of $r_{\text {drag }}$ provided by CMB data offers a tight constraint on the overall normalisation of the relation (see e.g., discussion in $[25,26]$ ). BAO measurements have been provided also for other surveys (e.g. Wigglez [27]), or for other subsamples (e.g. galaxy clusters, e.g. $[28,29])$, but since the covariance between these samples and the dataset used in this analysis has not been estimated, we decided not to use them.

Local $\boldsymbol{H}_{\mathbf{0}}$ value. Finally in some cases we also include the local distance ladder measurement of the Hubble constant $H_{0}=73.0 \pm 2.4 \mathrm{~km} / \mathrm{s} / \mathrm{Mpc}$, as measured by ref. [30] using the recalibration provided by ref. [31].

Since there have been claims of tensions between the local $H_{0}$ measurement and the value inferred from early-Universe observations within a $\Lambda$ CDM Universe (e.g., [10, 32-34] and references therein) when including CMB data we will always report results both with and without the $H_{0}$ measurement. In this way the reader can judge whether the inclusion of a possible "discrepant" measurement drives any of the conclusions.

The $\mathrm{SNe}$ and BAO data used in this analysis are shown in figure 2.

\section{Methodology}

Under the assumptions of isotropy and homogeneity, the metric of space-time of the Universe is fully specified by the Friedmann-Lemaitre- Robertson-Walker one. The most economic $\Lambda \mathrm{CDM}$ cosmological model can be described by six parameters, but three of them

\footnotetext{
${ }^{2}$ Anisotropic BAO measurements can measure $D_{\mathrm{A}}$ and $H(z)$ separately but at present the anisotropic measurements does not yet have significantly more statistical power than the isotropic one [17]. While ref. [10] for CMASS uses the anisotropic measurement from [17], here we use the isotropic one.
} 

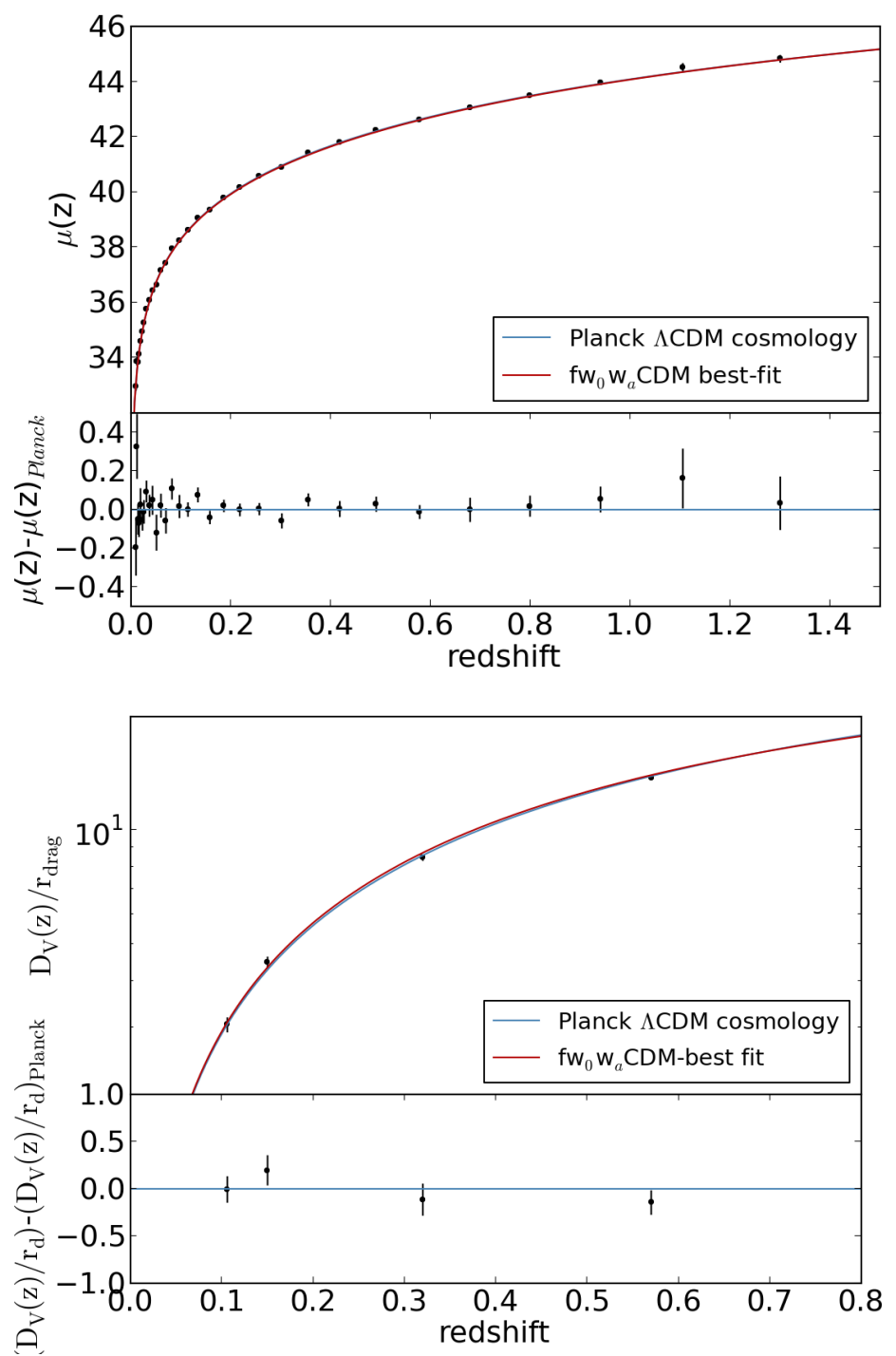

Figure 2. Same as figure 1, but for BAO and SNe datasets; SNe data have been taken from ref. [14], and BAO data from $[15-17]$.

univocally define the background evolution described by the Hubble parameter $H(z)=$ $H_{0} \sqrt{\Omega_{\mathrm{m}}(1+z)^{3}+\Omega_{\mathrm{DE}}}$. Extensions to this model have been proposed by relaxing one or more of its assumptions, such as flatness, dark energy Equation-of-State (EoS) parameter evolution, number of relativistic species in the Universe, total sum of neutrino masses; in this context, a generic model for the expansion rate of the Universe adopts a generic form for the equation of state parameter of dark energy $w(z)$, and is:

$$
H(z)=H_{0}\left\{\Omega_{r}(1+z)^{4}+\Omega_{m}(1+z)^{3}+\Omega_{k}(1+z)^{2}+\Omega_{\mathrm{DE}}(1+z)^{3\left(\int_{0}^{z} \frac{w\left(z^{\prime}\right)}{\left(1+z^{\prime}\right)} d z^{\prime}\right)}\right\}^{1 / 2},
$$

where $\Omega_{i}$ denote the energy density parameter for the various species in the Universe (matter, curvature, dark energy and radiation) at $z=0$. Given that the contribution to the total energy due to radiation is not significant at late time where $\Omega_{\mathrm{DE}}$ is important, we can safely neglect it; the relation between the energy density parameters is $1=\Omega_{m}+\Omega_{\mathrm{DE}}+\Omega_{k}$. 
A popular model for the expansion rate of the Universe is given by the CPL $[35,36]$ parameterisation of the equation of state for dark energy, $w(z)=w_{0}+w_{a}(z /(1+z))$, yielding for the expansion rate:

$$
H(z)=H_{0}\left\{\Omega_{r}(1+z)^{4}+\Omega_{m}(1+z)^{3}+\Omega_{k}(1+z)^{2}+\Omega_{\mathrm{DE}}(1+z)^{3\left(1+w_{0}+w_{a}\right)} e^{-3 w_{a} \frac{z}{1+z}}\right\}^{1 / 2} .
$$

The stronger dependence of $H(z)$ is on the Hubble constant, matter density and curvature parameter, while the dependence on dark energy EoS parameters is less significant, particularly for $w_{a}$ which is the most difficult parameter to constrain.

We follow two approaches. First, we analyze the constraints that can be put on cosmological parameters, and in particular on dark energy time evolution, with late-Universe probes (i.e. at $z<2$, not considering $\mathrm{CMB}$ data), such as $\mathrm{CC}$, $\mathrm{SNe}$ and $\mathrm{BAO}$ (with and without $H_{0}$ ), first separately then combined; then we explore how the constraints can be narrowed down by adding early-Universe information from CMB observations. Therefore, we will be able to test how the late and early Universe agrees with each other, being this fully determined within a given cosmological model, like $\Lambda$ CDM.

We analyze the goodness of fit of $\mathrm{CC}, \mathrm{SNe}$ and $\mathrm{BAO}$ with a standard $\chi^{2}$ approach; this is possible as the reported errors on the data are Gaussianly distributed. For SNe, we use the full covariance matrix, while for the other probes, measurements are uncorrelated and the covariance is diagonal; the $\chi^{2}$ have been estimated accordingly.

For $\mathrm{CC}$ we have that:

$$
\chi^{2}=\sum \frac{\left(H_{\mathrm{th}}(z)-H_{\mathrm{obs}}(z)\right)^{2}}{\sigma_{H_{\mathrm{obs}}}^{2}(z)},
$$

where $H_{\mathrm{th}}(z)$ is taken from eq. (3.2) and $H_{\mathrm{obs}}$ and $\sigma_{H_{\mathrm{obs}}}$ from ref. [3].

For SNe we have:

$$
\chi^{2}=\left(\mu_{b}-M-5 \log _{10}\left(D_{L}(z)\right)\right)^{t} C^{-1}\left(\mu_{b}-M-5 \log _{10}\left(D_{L}(z)\right)\right),
$$

where $D_{L}$ is the luminosity distance and $C$ the covariance matrix associated with distance modulus measurements $\mu_{b} .^{3}$

For BAO we have:

$$
\chi^{2}=\sum \frac{\left(D_{\mathrm{V}, \mathrm{th}}(z) / r_{\mathrm{drag}}-D_{\mathrm{r}, \mathrm{obs}}(z)\right)^{2}}{\sigma_{D_{\mathrm{r}, \mathrm{obs}}}^{2}(z)},
$$

where $D_{r}=D_{V} / r_{\text {drag }}$, and $D_{\mathrm{V}, \text { th }}$ is given by eq. (2.2). When CMB information is not included, $r_{\text {drag }}$ is treated as a nuisance parameter i.e., marginalised over.

We sample the distribution of parameters with the public python package emcee [37], which is an implementation of the affine-invariant ensemble sampler for Markov chain Monte Carlo (MCMC) proposed by ref. [38]. Most uses of MCMC in the astrophysics literature are based on slight modifications to the Metropolis-Hastings method. The performance of this sampler is very sensitive to the choice of the proposal distribution and in particular its covariance matrix and there is no easy and straightforward method to optimize this. However, the affine-invariant ensemble sampling algorithm is much more efficient and does not depend on the aspect ratio in highly anisotropic distributions. The method evolves simultaneously an

\footnotetext{
${ }^{3}$ http://supernovae.in2p3.fr/sdss_snls_jla/ReadMe.html.
} 
ensemble of many $(K \gg 10)$ walkers where the proposal distribution for one walker $k$ is based on the current positions of the $K-1$ walkers. To update the position of a walker at position $X_{k}$, a walker $X_{j}$ is drawn randomly from the remaining walkers and its new position will be a step away from the position of walker $X_{j}$ with a specific proposal distribution with one tunable parameter ("stretch move"). This approach can be further tuned to be parallelized.

We considered uniform priors on the following variables: $H_{0}=[50,100] \mathrm{km} / \mathrm{s} / \mathrm{Mpc}$, $\Omega_{m}=[0.01,0.99], w_{0}=[-3,0], w_{a}=[-5,5], r_{\text {drag }}=[100-200]$.

We first explore a flat model $\left(\Omega_{k}=0\right)$ with the CPL parameterisation for $w(z)$ $\left(\mathrm{fw}_{0} w_{a} \mathrm{CDM}\right.$ hereafter) to compare the results with the ones that can be obtained from CMB [10].

Subsequently, to include early time (CMB) constraints we have used the posterior samples provided by the Planck 2015 data release, and importance sampled them with $\mathrm{CC}$ measurements, comparing the results with the ones obtained from the combination of Planck15+BAO and Planck15+SNe provided by the Planck team [10]. The joint posterior for two data sets $\left(D_{1}\right.$ and $\left.D_{2}\right)$ can be estimated easily if the posterior for e.g. $D_{1}$ has already been simulated with an MCMC. The weight of each point in the chain is proportional to the posterior for $D_{1}$ which is the product of the likelihood for $D_{1}$ and the prior. The joint posterior for $D_{1}$ and $D_{2}$ is proportional to the product of the likelihood for $D_{1}$, the likelihood for $D_{2}$ and the prior, which can be obtained by reweighting the weight of the chain by the likelihood of $D_{2}$. This procedure is called importance sampling. The simulated posterior so obtained is a good approximation if data set $D_{2}$ is consistent with $D_{1}$.

\section{Results}

We start by discussing the results obtained from late-Universe probes: cosmic chronometer analysis alone, and then in combination with other probes (BAO, SNe). We then combine late-Universe probes with early-Universe observations (CMB) to improve constraints also on parameters that do not have a direct effect on $H(z)$, such as the number of the relativistic species in the Universe $N_{\text {eff }}$ and the sum of the neutrino masses $\Sigma m_{\nu}$, by breaking parameter degeneracies.

\subsection{Constraints from the late-Universe probes}

Figure 3 shows constraints on a flat $w_{0} w_{a} \mathrm{CDM}$ cosmology using $\mathrm{CC}, \mathrm{SNe}, \mathrm{BAO}$ individually and their combination. Marginalised constraints for each parameter are reported in table 1. From this analysis, it is evident that $\mathrm{CC}$ and $\mathrm{BAO}$ have a similar constraining power in the $w_{0}-w_{a}$ plane, with CC providing better constraints on $H_{0}\left(H_{0}=73.2_{-8.1}^{+10}\right)$ and slightly better constraints on $\Omega_{m}$; SNe, while insensitive to $H_{0}$, provide stringent constraints on the dark energy EoS evolution. The poor constraining power on $H_{0}$ of the BAO analysis results from the fact that $r_{\mathrm{drag}}$ is treated as a nuisance parameter (i.e. is left as a free parameter, without any CMB prior, and is marginalised over). Most interestingly, the directions of the degeneracy between parameters in the various probes are different, and hence their combination can provide more stringent constraints, as shown by figure 3 bottom right panel. More quantitatively, the constraints obtained with $\mathrm{CC}$ are $\sim 70 \%$ and $\sim 50 \%$ better than the ones from BAO respectively for $H_{0}$ and $\Omega_{m}$, while just $\sim 8 \%$ for the dark energy EoS parameters. The constraints from $\mathrm{SNe}$ are better than the ones for CC of a factor $\sim 3.5$ for $w_{0}$, and of $\sim 60 \%$ for $w_{a}$, while being worst by $\sim 50 \%$ on $\Omega_{m}$. The combination of all late-Universe probes yields to a significant improvement of $\sim 40 \%$ on $\Omega_{m}$ with respect to SNe alone, and helps 

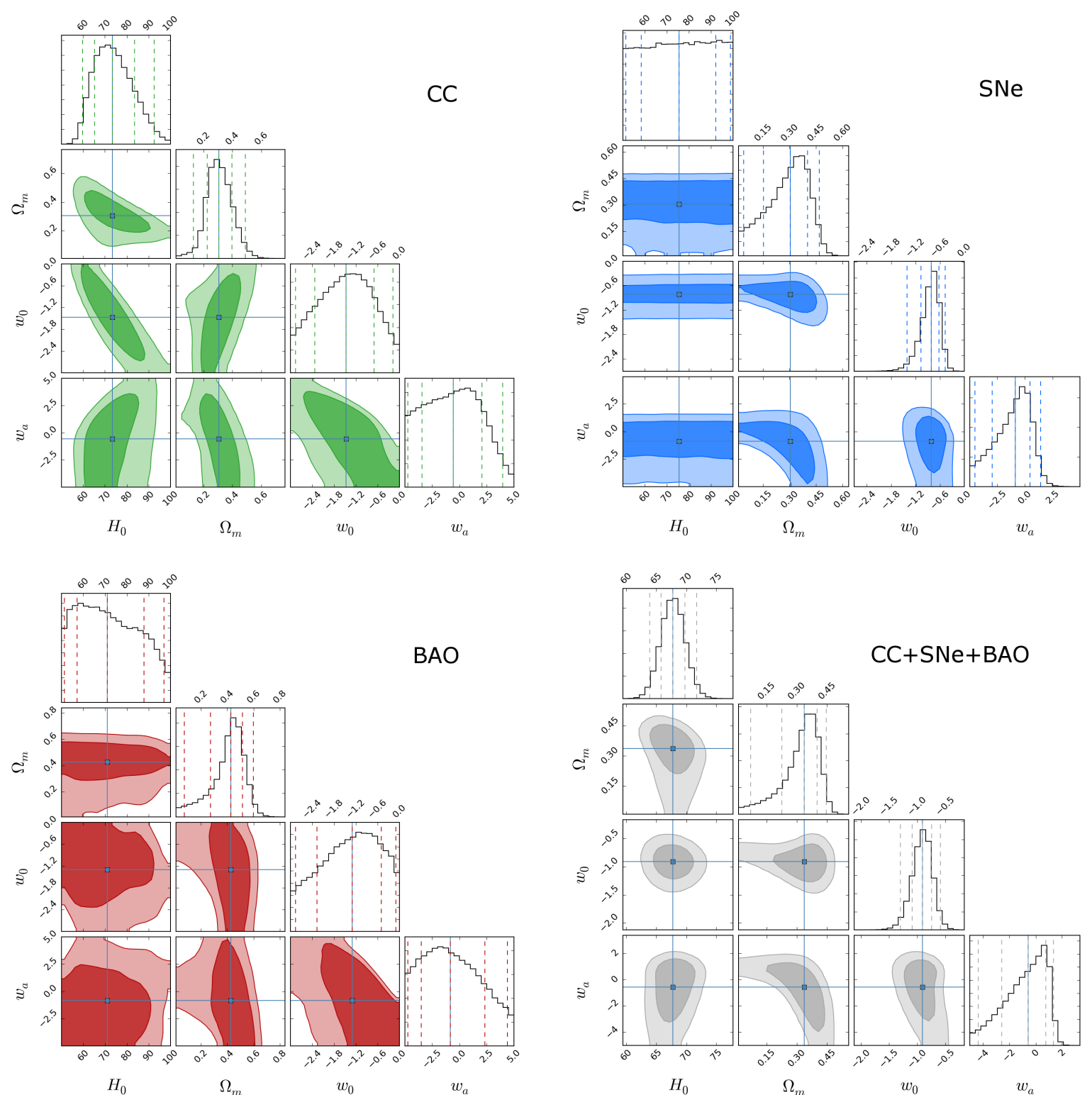

Figure 3. Constraints on a flat $w_{0} w_{a}$ CDM cosmology from cosmic chronometers (in green, upper-left panel), $\mathrm{SNe}$ (in blue, upper-right panel), BAO data (in red, lower-left panel), and from the combination of the various probes (in grey, lower-right panel) obtained with a MCMC approach. In each panel the contour plots are shown at $68 \%$ and $95 \%$ confidence level, and the posterior distribution of $\mathrm{H}_{0}, \Omega_{m}$, $w_{0}$ and $w_{a}$, with the $68 \%$ and $95 \%$ confidence level limits.

to partially remove the degeneracy in the $w_{0}-w_{a}$ plane, improving by $\sim 25 \%$ the measurements of $w_{0}$. In particular, it is interesting to note the orthogonality between the constraints provided by $\mathrm{CC}$ and SNe. We actually verified that most of the constraining power of the combination $\mathrm{CC}+\mathrm{SNe}+\mathrm{BAO}$ comes from $\mathrm{CC}+\mathrm{SNe}$, as they provide constraints for most parameters almost as stringent as when $\mathrm{BAO}$ data is added. For $w_{a}$, which is the more difficult parameter to be constrained, the addition of $\mathrm{BAO}$ proves to be helpful to reduce errorbars.

In order to gain further insights into the time evolution of dark energy, it is useful to explore beyond the two-parameter $w_{0}-w_{a}$ fit. Ideally, one would like to reconstruct $w(z)$ in 


\begin{tabular}{|ccccc|}
\hline \multicolumn{5}{|c|}{$\begin{array}{c}\text { MARGINALIZED 1D CONSTRAINTS } \\
\text { f } w_{0} w_{a} \text { CDM model }\end{array}$} \\
\hline & $\mathrm{H}_{0}$ & $\Omega_{\mathrm{m}}$ & $w_{0}$ & $w_{a}$ \\
\hline $\mathrm{CC}$ & $73.2_{-8.1}^{+10}$ & $0.31_{-0.08}^{+0.09}$ & $-1.46_{-0.87}^{+0.77}$ & $-0.5_{-2.9}^{+2.6}$ \\
$\mathrm{SNe}$ & - & $0.31_{-0.15}^{+0.1}$ & $-0.82_{-0.26}^{+0.19}$ & $-0.9_{-2.1}^{+1.3}$ \\
$\mathrm{BAO}$ & $70_{-14}^{+17}$ & $0.42_{-0.16}^{+0.09}$ & $-1.3_{-0.97}^{+0.81}$ & $-0.8_{-2.7}^{+3.2}$ \\
\hline $\mathrm{CC}+\mathrm{SNe}$ & $68.2_{-2}^{+2.1}$ & $0.34_{-0.1}^{+0.06}$ & $-0.9_{-0.19}^{+0.17}$ & $-1.1_{-2.1}^{+1.7}$ \\
$\mathrm{CC}+\mathrm{SNe}+\mathrm{BAO}$ & $67.8 \pm 2$ & $0.33_{-0.12}^{+0.06}$ & $-0.9_{-0.19}^{+0.17}$ & $-0.5_{-2}^{+1.3}$ \\
\hline
\end{tabular}

Table 1. Constraints on $\mathrm{H}_{0}, \Omega_{\mathrm{m}}, w_{0}$ and $w_{a}$ (68\% confidence limits) obtained for a flat $\Lambda$ CDM cosmology with equation-of-state parameter for dark energy parameterised as $w(z)=w_{0}+w_{a}(z /(1+z))$.

a non-parametric way; unfortunately, this is not possible (e.g. see ref. [11]). So, alternatively, one needs to use a parameterisation that is the least possible model dependent. Ref. [11] proposed an expansion with Chebyshev polynomials, $T_{i}$, without any further assumptions on the shape of the dark energy EoS evolution:

$$
w(z)=\sum_{i=0}^{N} \omega_{i} T_{i}(x(z))
$$

where $T_{i}(x)$ are the Chebyshev polynomials: up to the second order $T_{0}=1, T_{1}=x, T_{2}=$ $2 x^{2}-1, x=\left(2 z / z_{\max }\right)-1$ and $\omega_{i}$ are the parameters to be constrained by the data.

In this framework, it is possible to write the expansion history (for a flat Universe) as:

$$
H(z)=H_{0}(1+z)^{3 / 2} \sqrt{\Omega_{m}+\left(1-\Omega_{m}\right) \exp \left(\frac{3}{2} z_{\max } \sum_{i=0}^{N} \omega_{i} G_{i}(z)\right)}
$$

where the functions $G_{i}$ can be obtained iteratively from the equation $J_{i}=1 / n b\left[\left(2 z / z_{\max }-\right.\right.$ $\left.1)^{i}-(-1)^{i}\right]-(a / b) J_{i-1}, J_{0}=1 / b \log (1+z), a=1+\left(z_{\max } / 2\right)$ and $b=z_{\max } / 2$. Up to the first two orders, it can be obtained that $G_{0}=J_{0}, G_{1}=J_{1}$ and $G_{2}=2 J_{2}-J_{0}$ [11].

In table 2 we report the results on the constraints of $\omega_{i}$ up to both first and second order obtained by fitting jointly $\mathrm{CC}, \mathrm{SNe}$ and $\mathrm{BAO}$ data to eq. (4.2), and in figure 4 we show the contours for the fit up to the second order. Because of the increased freedom in the EoS parameterisation, in this analysis we always include the $H_{0}$ measurement.

The present-day value of $w$ in this expansion can be estimated as:

$$
w_{z=0}=\sum_{i=0}^{N}(-1)^{i} \omega_{i}
$$

from which we find from the combined constraints $w_{z=0}=-1.21_{-0.81}^{+0.69}$ with the first order, and $w_{z=0}=-1.5_{-1.56}^{+1.4}$ with the second order expansion.

In this analysis by combining $\mathrm{SNe}$ and $\mathrm{BAO}$ data with the latest $\mathrm{CC}$ measurements (and the $H_{0}$ prior), we are able to close for the first time the $2 \sigma$ contours on the $\omega_{i}$ parameters up 


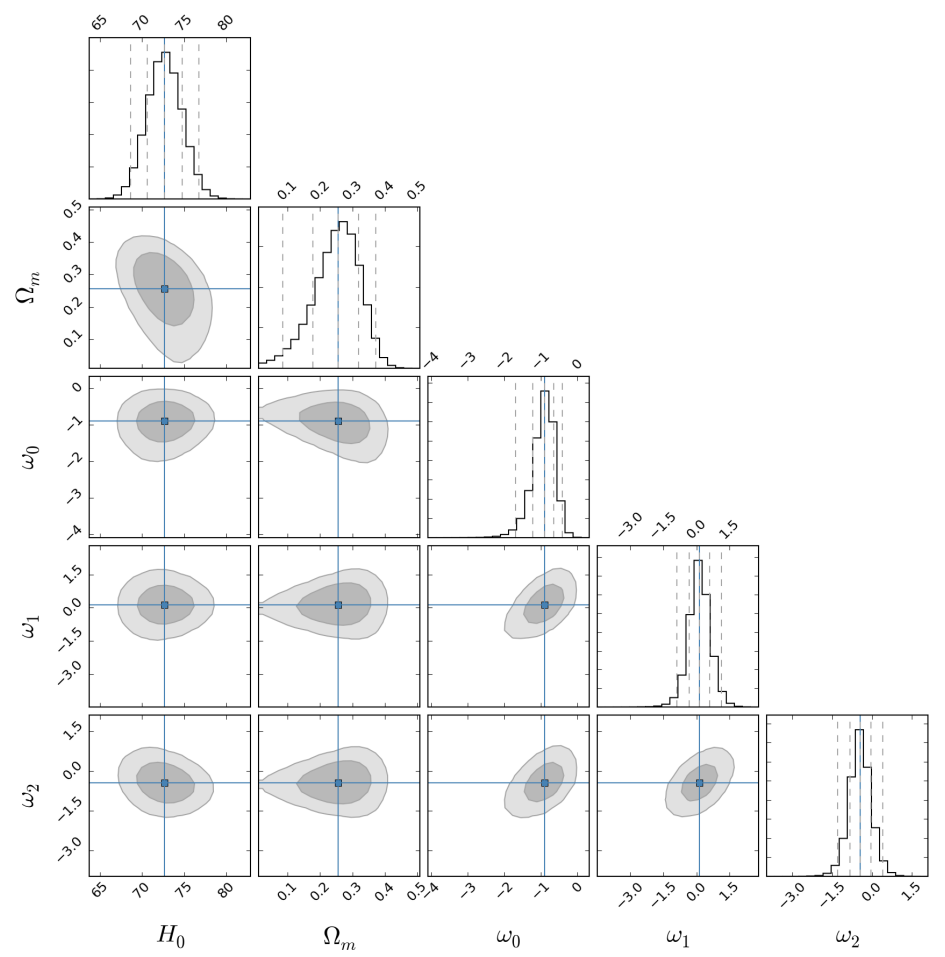

Figure 4. Constraints for the Chebyshev expansion fit obtained from the combination of cosmic chronometer, SNe and BAO data. In each panel the contour plots are shown at $68 \%$ and $95 \%$ confidence level, and the posterior distribution of $\mathrm{H}_{0}, \Omega_{m}, \omega_{0}, \omega_{1}$ and $\omega_{2}$, with the $68 \%$ and $95 \%$ confidence level limits. For these constraints we assumed a Gaussian prior on $H_{0}=73 \pm 2.4[30,31,39]$.

\begin{tabular}{|cccccc|}
\hline \multicolumn{5}{c|}{$\begin{array}{c}\text { MARGINALIZED 1D CONSTRAINTS } \\
\text { Chebyshev expansion }\end{array}$} \\
\hline & $\mathrm{H}_{0}$ & $\Omega_{\mathrm{m}}$ & $\omega_{0}$ & $\omega_{1}$ & $\omega_{2}$ \\
\hline $\mathrm{CC}+\mathrm{H}_{0}$ & - & $0.36 \pm 0.04$ & $-7.6_{-6.5}^{+6.2}$ & $-6.1_{-9.3}^{+8.7}$ & $-0.2_{-3.5}^{+6.3}$ \\
\hline $\mathrm{CC}+\mathrm{SNe}+\mathrm{BAO}+\mathrm{H}_{0}-1^{\text {st }}$ order & - & $0.27_{-0.06}^{+0.05}$ & $-0.68_{-0.21}^{+0.16}$ & $0.53_{-0.21}^{+0.30}$ & - \\
$\mathrm{CC}+\mathrm{SNe}+\mathrm{BAO}-2^{\text {nd }}$ order & $71.9_{-3.9}^{+4.2}$ & $0.27_{-0.1}^{+0.08}$ & $-0.91_{-0.36}^{+0.27}$ & $0.12_{-0.49}^{+0.49}$ & $-0.43_{-0.43}^{+0.51}$ \\
$\mathrm{CC}+\mathrm{SNe}+\mathrm{BAO}+\mathrm{H}_{0}-2^{\text {nd }}$ order & - & $0.26_{-0.08}^{+0.06}$ & $-0.91_{-0.33}^{+0.26}$ & $0.13_{-0.45}^{+0.47}$ & $-0.46_{-0.39}^{+0.4}$ \\
\hline
\end{tabular}

Table 2. Constraints on $\mathrm{H}_{0}, \Omega_{\mathrm{m}}$, and $\omega_{0}, \omega_{1}$ and $\omega_{2}$ parameters (from eq. (4.1)) at $68 \%$ confidence level obtained from the fit to the Chebyshev expansion of $w(z)$ up to the second order as modeled in eq. (4.2). When a Gaussian prior on $H_{0}$ is assumed $\left(H_{0}=73 \pm 2.4[30,31,39]\right)$, no constraints are provided for $H_{0}$, because we will be simply recovering the prior.

to the second order, significantly improving on previous measurements [11] where unbounded constraints were obtained, and only up to the first order. This is an important result, since it provides a measurement of the evolution of dark energy EoS with a completely independent parameterisation. To check whether the results are being affected by the inclusion of the prior on $H_{0}$, we performed the analysis also without adding it, finding no significant differ- 


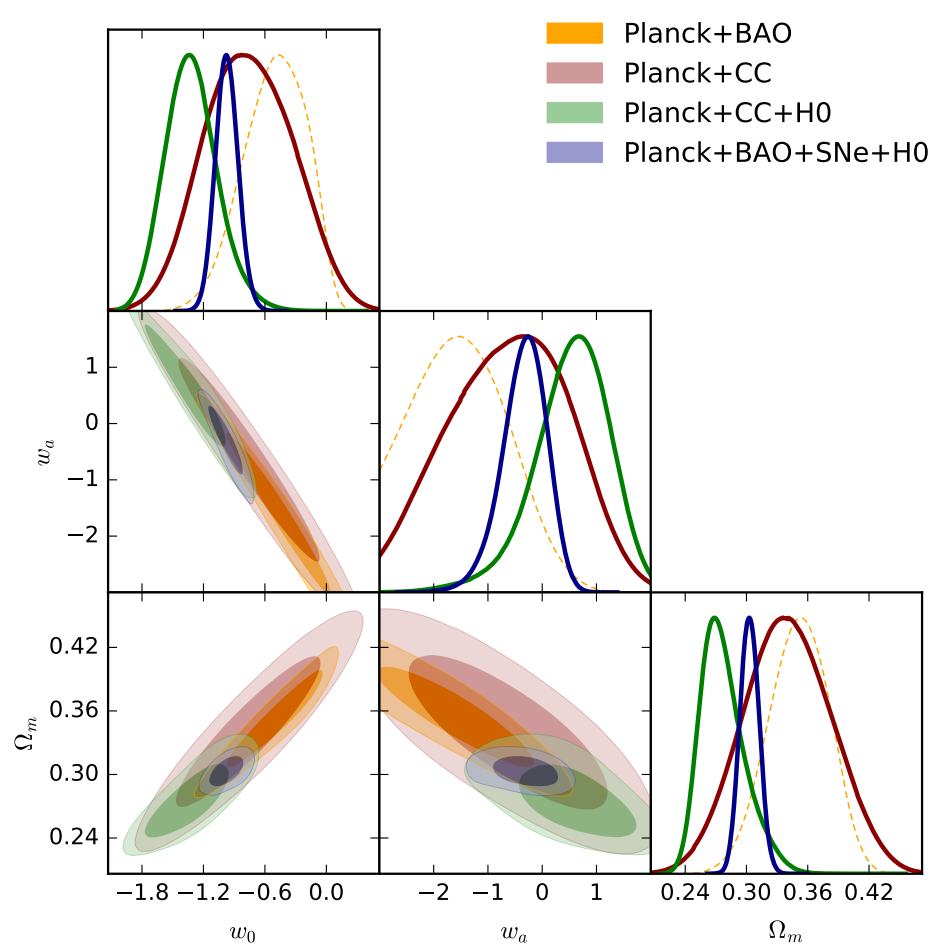

Figure 5. Constraints on $\Omega_{\mathrm{m}}, w_{0}$ and $w_{a}$ for a f $w_{0} w_{a}$ CDM model obtained with different combinations of data sets. Cosmic chronometers and BAO have a similar constraining power in the $w_{0}-w_{a}$ plane, as shown in figure 3, and the combination of $\mathrm{CMB}+\mathrm{BAO}$ and $\mathrm{CMB}+\mathrm{CC}$ provides comparable results. We used $H_{0}=73 \pm 2.4 \mathrm{~km} / \mathrm{s} / \mathrm{Mpc}$ from refs. [30, 31, 39].

ences, but, as expected, larger errorbars; these results are reported in table 2. In figure 6 we compare the estimated evolution of $w(z)$ with different parameterisations and different datasets combinations, finding, remarkably, results in very good agreement, and compatible with a cosmological constant. We note that $w(z)$ in the Chebyshev expansion appears to slightly deviate from a cosmological constant at the highest redshifts $(z \sim 2)$; this deviation, however, is not significant, and well below two sigma.

\subsection{Combining early-Universe information}

$\mathrm{CC}$ measurements are extremely useful in breaking CMB parameters degeneracies for models beyond the "minimal" $\Lambda$ CDM model. In particular we will show their fundamental importance in models that for allow a time-variability of the dark energy equation of state and for models where CMB constraints show parameter degeneracies with the expansion history.

We start extending the "minimal" $\Lambda \mathrm{CDM}$ where the additional parameter affects directly the late-time expansion history. We first explore a flat $w_{0} w_{a} \mathrm{CDM}$ model which constraints are presented in figure 5 and table 3. For the $\mathrm{f} w_{0} w_{a} \mathrm{CDM}$ model, the base analysis provided by the Planck collaboration include Planck15 and BAO data; as demonstrated in section 4.1, CC and BAO have a similar constraining power on the $w_{0}-w_{a}$ plane, hence when combining Planck15+BAO+CC we obtain a little gain in the constraints, still however being able to close the $95 \%$ confidence level contours for $w_{a}$. When including also $H_{0}$, the errorbars shrink considerably, and we obtain $w_{0}=-1.09_{-0.21}^{+0.19}$ and $w_{a}=-0.02_{-0.49}^{+0.61}$ (at $68 \%$ confidence level); in comparison, the combination Planck15+BAO $+\mathrm{CC}+\mathrm{SNe}+\mathrm{H}_{0}$ obtains $w_{0}=-0.98 \pm 0.11$ and $w_{a}=-0.30_{-0.34}^{+0.42}$ (at $68 \%$ confidence level). 


\begin{tabular}{|c|c|c|c|}
\hline \multicolumn{4}{|c|}{$\begin{array}{l}\text { MARGINALIZED 1D CONSTRAINTS } \\
\text { f } w_{0} w_{a} \text { CDM model }\end{array}$} \\
\hline & $\Omega_{\mathrm{m}}$ & $w_{0}$ & $w_{a}$ \\
\hline Planck15+BAO & $0.351_{-0.028}^{+0.032}$ & $-0.51_{-0.26}^{+0.36}$ & $-1.47_{-1.0}^{+0.78}$ \\
\hline Planck15+CC & $0.314_{-0.052}^{+0.039}$ & $-0.81 \pm 0.40$ & $-0.8_{-1.0}^{+1.2}$ \\
\hline Planck15 $+\mathrm{CC}+H_{0}$ & $0.273_{-0.020}^{+0.017}$ & $-1.18_{-0.25}^{+0.20}$ & $0.01_{-0.44}^{+0.63}$ \\
\hline Planck15 $+\mathrm{BAO}+\mathrm{CC}+H_{0}$ & $0.294_{-0.018}^{+0.016}$ & $-1.09_{-0.21}^{+0.19}$ & $-0.02_{-0.49}^{+0.61}$ \\
\hline Planck15 $+\mathrm{BAO}+\mathrm{SNe}+H_{0}$ & $0.3031 \pm 0.0093$ & $-0.97 \pm 0.11$ & $-0.34_{-0.35}^{+0.45}$ \\
\hline Planck15 $+\mathrm{BAO}+\mathrm{CC}+\mathrm{SNe}+H_{0}$ & $0.3032 \pm 0.0092$ & $-0.98 \pm 0.11$ & $-0.30_{-0.34}^{+0.42}$ \\
\hline
\end{tabular}

Table 3. Marginalised constraints on $\Omega_{\mathrm{m}}, \Omega_{\mathrm{DE}}, \Omega_{\mathrm{k}}, w_{0}$ and $w_{a}$ at $68 \%$ confidence level obtained for a flat $\Lambda \mathrm{CDM}$ cosmology with equation-of-state parameter for dark energy parametrized as $w(z)=$ $w_{0}+w_{a}(z /(1+z))\left(\mathrm{f} w_{0} w_{a} \mathrm{CDM}\right)$. We adopted $H_{0}=73 \pm 2.4 \mathrm{~km} / \mathrm{s} / \mathrm{Mpc}$ from refs. [30, 31, 39].

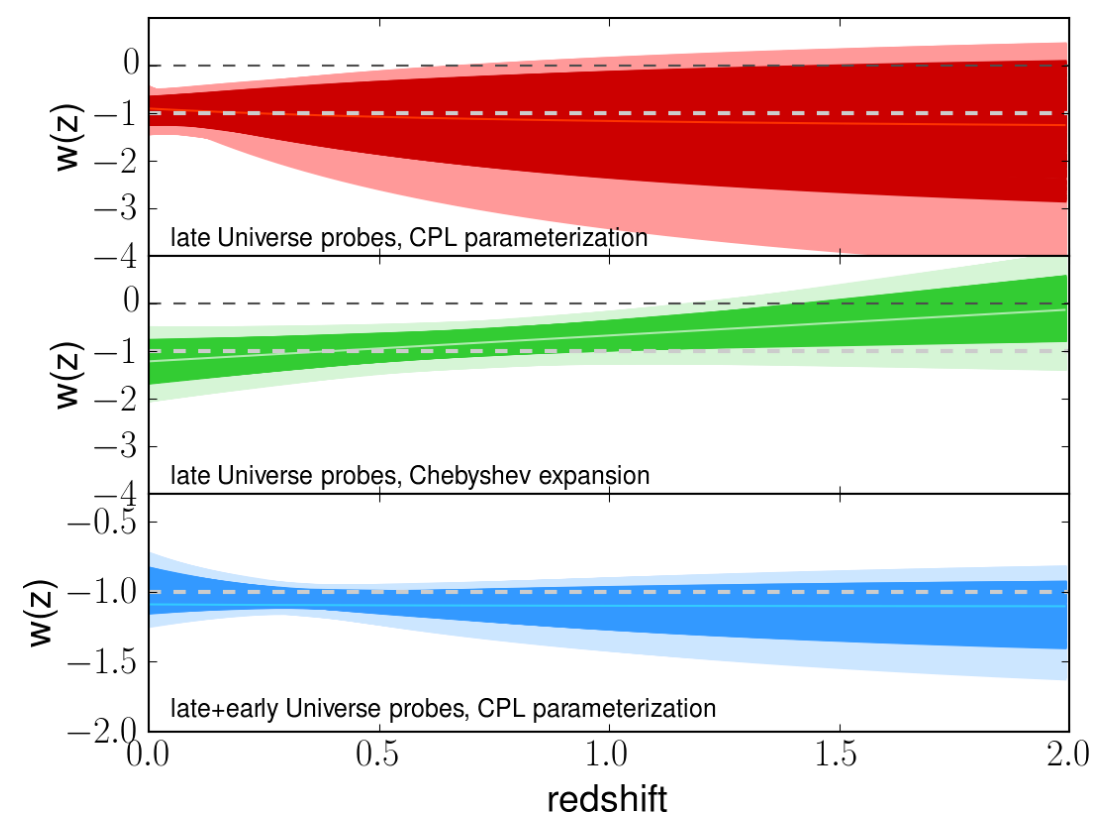

Figure 6. Reconstruction of the time evolution of dark energy EoS obtained from different probes. Red contours the constraints obtained from late-Universe probes with a CPL parameterisation (see eq. (3.2) and table 1), green contours show the constraints obtained from late-Universe probes with Chebyshev decomposition up to the first order (see eq. (4.1) and table 2), and blue contours the constraints obtained adding also CMB information with the CPL parameterisation (see table 3). The darker regions show in each case the $68 \%$ confidence level contours, while the light ones the $95 \%$ confidence level contours. These constraints assume $H_{0}=73 \pm 2.4 \mathrm{~km} / \mathrm{s} / \mathrm{Mpc}$, taken from refs. $[30,31,39]$.

In figure 6 we show the evolution of $w(z)$ up to $z=2$ (which is the maximum redshift covered by our datasets) for different parameterisations. We compare the constraints obtained from Chebyshev reconstruction and from a CPL parameterisation from late-Universe 


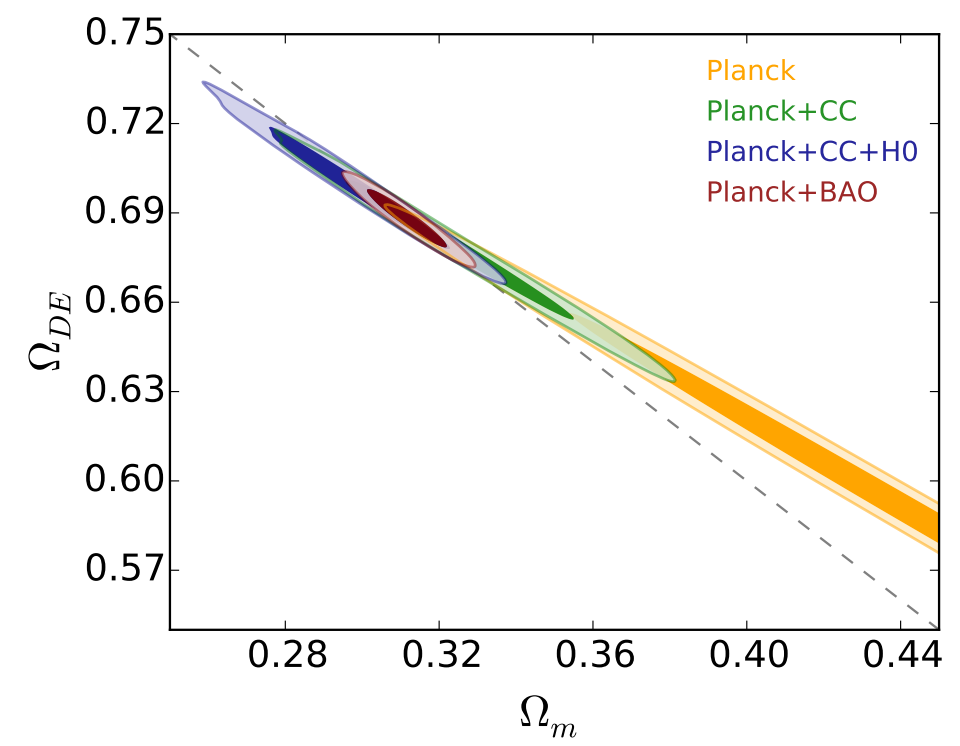

Figure 7. Constraints in the $\Omega_{\mathrm{DE}}-\Omega_{\mathrm{m}}$ plane for different data-sets combinations. Note that combining cosmic chronometer data-set with CMB data helps in constraining the contours close to the $\Omega_{\mathrm{k}}=0$ region, indicated with the dashed line. Similar results are obtained when combining the BAO with the CMB. We used $H_{0}=73 \pm 2.4 \mathrm{~km} / \mathrm{s} / \mathrm{Mpc}$ from refs. [30, 31, 39].

probes alone, and from a CPL parameterisation combining also Planck15 measurements. For homogeneity with CPL, we show here the results from Chebyshev reconstruction up to the first order, to compare results with similar degrees of freedom. We find that all the best-fit constraints show deviations from $\Lambda \mathrm{CDM}$ only up to $\sim 25 \%$ up to $z=2$ for the CPL parameterization, and up to $\sim 60 \%$ up to $z=1.5$ for the Chebyshev reconstruction. Quite remarkably, all measurements are compatible at $2 \sigma$ with a constant dark energy EoS $w=-1$ in the entire redshift range. The most accurate measurement is obtained when combining both late- and early-Universe probes together, providing a constraint on $w(z)$ which is consistent with the $\Lambda \mathrm{CDM}$ model at the $40 \%$ level over the entire redshift range $0<z<2$ (at $68 \%$ confidence level).

We then consider an open $\Lambda \mathrm{CDM}$ model, to explore the constraints that can be obtained on curvature from the different probes. The results are shown in figure 7 and table 4 . We find that cosmic chronometer are fundamental to break the $\Omega_{\mathrm{m}}-\Omega_{\mathrm{DE}}$ degeneracy present in CMB only data, constraining $\Omega_{k}=-0.0030_{-0.0042}^{+0.0055}$. The error bars can be reduced when also the $H_{0}$ data is included, obtaining $\Omega_{k}=0.0028_{-0.0031}^{+0.0035}$. For comparison, BAO data provide a similar result, $\Omega_{k}=0.0002 \pm 0.0021$.

It is possible to constrain neutrino properties from the combination of CMB data with late-Universe measurement of the expansion rate (e.g., see refs. [30, 40, 41]). In this work, we consider a simple flat $\Lambda$ CDM Universe where the effective number of relativistic species $N_{\text {eff }}$ is let free, and not fixed to the standard value of $N_{\text {eff }}=3.04$; we analyze this model studying the combined dataset of Planck15 and $\mathrm{CC}$ and $H_{0}$.

Results are shown in figure 8 and table 5 . Planck $15+\mathrm{CC}+H_{0}$ have the same constraining power as Planck15+BAO. They restrict $N_{\text {eff }}=3.17_{-0.30}^{+0.29}$. Clearly, CC are not able to measure $N_{\text {eff }}$ directly, but provide constraints by breaking the degeneracy between the number of relativistic species and the parameters that fix the matter-radiation equality and the expansion history (e.g., see ref. [41]). We then fit a flat $\Lambda$ CDM Universe where the total neu- 


\begin{tabular}{|c|c|c|c|}
\hline \multicolumn{4}{|c|}{$\begin{array}{l}\text { MARGINALIZED 1D CONSTRAINTS } \\
\text { o } \Lambda \text { CDM model }\end{array}$} \\
\hline & $\Omega_{\mathrm{m}}$ & $\Omega_{\mathrm{DE}}$ & $\Omega_{k}$ \\
\hline Planck15 & $0.47_{-0.086}^{+0.063}$ & $0.57_{-0.047}^{+0.064}$ & $-0.040_{-0.016}^{+0.023}$ \\
\hline Planck15+CC & $0.325_{-0.023}^{+0.018}$ & $0.678_{-0.015}^{+0.018}$ & $-0.0030_{-0.0042}^{+0.0055}$ \\
\hline Planck15 $+\mathrm{CC}+\mathrm{H}_{0}$ & $0.298 \pm 0.015$ & $0.699 \pm 0.013$ & $0.0028_{-0.0031}^{+0.0035}$ \\
\hline Planck15+BAO & $0.3117 \pm 0.0070$ & $0.6881 \pm 0.0065$ & $0.0002 \pm 0.0021$ \\
\hline
\end{tabular}

Table 4. Constraints on $\Omega_{\mathrm{m}}, \Omega_{\mathrm{DE}}$ and $\Omega_{k}$ at $68 \%$ confidence level obtained for an open $\Lambda \mathrm{CDM}$ cosmology. For $H_{0}$, we considered the value $73 \pm 2.4 \mathrm{~km} / \mathrm{s} / \mathrm{Mpc}$ from refs. [30, 31, 39].

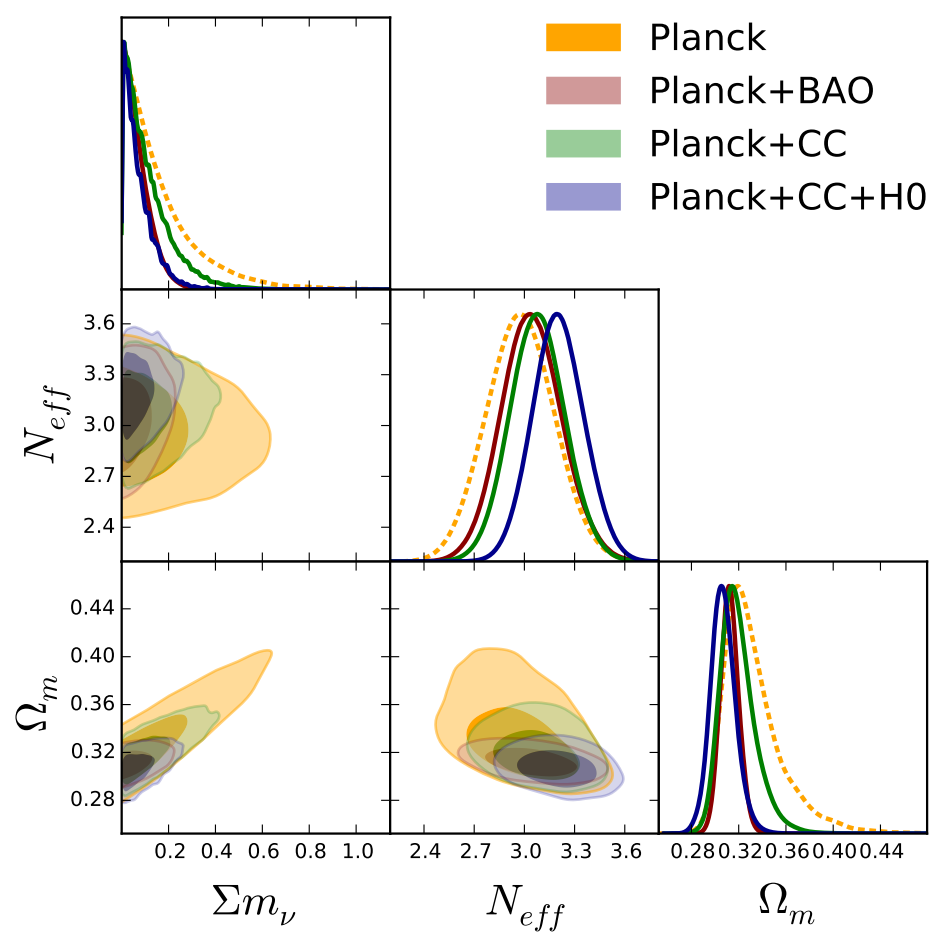

Figure 8. Constraints on number of effective relativistic species $N_{\text {eff }}$ and their total mass $\Sigma m_{\nu}$ obtained with different combination of probes. Note that cosmic chronometers data-set in combination with the $\mathrm{CMB}$ provide comparable constraints to those of $\mathrm{CMB}+\mathrm{BAO}$. We adopted $H_{0}=73 \pm$ $2.4 \mathrm{~km} / \mathrm{s} / \mathrm{Mpc}$ from refs. [30, 31, 39].

trino mass is left as a free parameter, and looked at the constraints on the sum of neutrino masses. ${ }^{4}$ We obtain a constraint of $M_{\nu}<0.27$ at $95 \%$ confidence. We note how the addition of CC data helps to significantly reduce the errorbars both on neutrino mass and $N_{\text {eff }}$ with respect to Planck data alone.

Finally, to exploit the constraining power of our new results in the $w_{0}-w_{a}$ plane, in figure 9 we compare our measurements with some theoretical models taken from refs. [36, 42].

\footnotetext{
${ }^{4}$ We assume three degenerate neutrino species; current data have no sensitivity on the hierarchy, so this is a very good approximation.
} 


\begin{tabular}{|c|c|c|}
\hline \multicolumn{3}{|c|}{$\begin{array}{l}\text { MARGINALIZED }(95 \%) 1 \mathrm{D} \text { CONS } \\
\qquad \Lambda \mathrm{CDM}+\Sigma m_{\nu} \text { model }\end{array}$} \\
\hline & $\Sigma m_{\nu}$ & $N_{\text {eff }}$ \\
\hline Planck15 & $<0.577$ & $2.93_{-0.38}^{+0.39}$ \\
\hline Planck15+CC & $<0.423$ & $3.05_{-0.32}^{+0.33}$ \\
\hline Planck15+CC $+H_{0}$ & $<0.269$ & $3.17_{-0.30}^{+0.29}$ \\
\hline Planck15+BAO & $<0.222$ & $2.99_{-0.34}^{+0.37}$ \\
\hline
\end{tabular}

Table 5. Constraints on $\Sigma m_{\nu}$ (in units of eV) and $N_{\text {eff }}$ at $95 \%$ confidence level obtained for a flat $\Lambda \mathrm{CDM}$ cosmology. For the Planck $15+\mathrm{CC}+H_{0}$ combination, we assumed a Gaussian prior $73 \pm$ $2.4 \mathrm{~km} / \mathrm{s} / \mathrm{Mpc}$ from refs. [30, 31, 39].

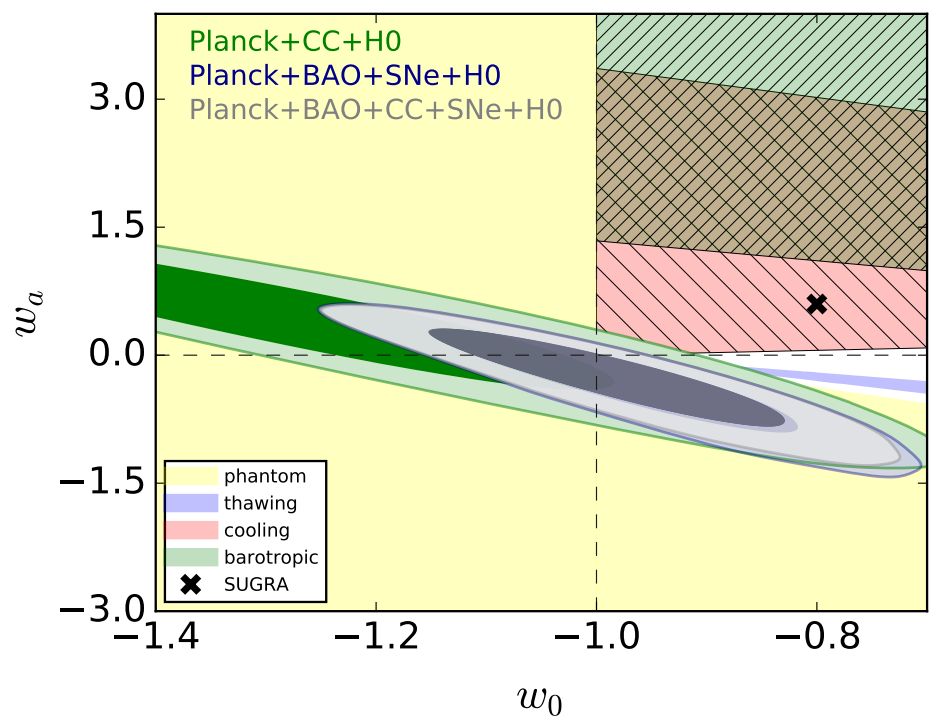

Figure 9. Constraints on the time evolution of dark energy equation of state compared to several theoretical models (the brown region is the overlap between barotropic and cooling quintessence models). Models have been taken from refs. [36, 42]. Dashed lines correspond to the position of the cosmological constant, $\left(w_{0}, w_{a}\right)=(-1,0)$. The Hubble constant considered for these constraints is $H_{0}=73 \pm 2.4 \mathrm{~km} / \mathrm{s} / \mathrm{Mpc}$ from refs. [30, 31, 39].

They comprise a large variety of dark energy models, including barotropic models, phantom models, two different quintessence models and a SUGRA model. We refer to refs. [42, 43] for a comprehensive description of the models. Here we note that thank to the newly analysed data, the $95 \%$ allowed region in the $w_{0}-w_{a}$ plane is significantly reduced. The new constraints allow not only to reject at $95 \%$ confidence level the barotropic models, but also to exclude almost all quintessence models and the SUGRA model.

\section{Conclusions}

Measurements of the expansion history are the only direct indication that the Universe is undergoing an accelerated expansion, therefore suggesting that it should be dominated by 
a dark energy component [3]. Given the magnitude of the implications of the accelerated expansion, it is important to have different, independent and complementary observations of the expansion history.

In this work we have used the latest cosmic chronometers measurements to set constraints on the time evolution of dark energy. We have shown that cosmic chronometers alone are able to set constraints on cosmological parameters comparable to other state-ofthe-art late-Universe probes, namely BAO and SNe. However, unlike BAO and SNe, the cosmic chronometer data provide a direct measurement of the expansion rate $H(z)$, without having to assume a cosmological model, and thus can be used to test it. In particular, the constraints on cosmological parameters from cosmic chronometers and SNe demonstrated to be almost orthogonal one to another. Furthermore, their joint constraints prove to be as stringent as those obtained adding BAO data.

Remarkably, we find that constraints on dark energy are fully consistent with a cosmological constant up to $z \sim 2$, and when combining late- and early-Universe probes we find deviations from the $\Lambda \mathrm{CDM}$ model smaller than $40 \%$ over the entire redshift range $0<z<2$ (at $68 \%$ confidence level). We tested these results considering two independent parameterizations, the standard CPL parameterization and an expansion of the dark energy EoS based on Chebyshev polynomials. The conclusions proved to be stable to the assumed parameterisation for $w(z)$.

In the CPL parameterisation, from the combination of late-Universe probes alone we find $w_{0}=-0.9 \pm 0.18$ and $w_{a}=-0.5 \pm 1.7$, and when combining also CMB measurements from Planck15 we obtain $w_{0}=-0.98 \pm 0.11$ and $w_{a}=-0.30 \pm 0.4$. We compared these constraints with a set of theoretical dark energy models, finding that quintessence is disfavoured by the data at high significance, and providing strong constraints on the allowed families of models. We also tightly constrain the geometry of the Universe, obtaining a density parameter for curvature $\Omega_{k}=0.003 \pm 0.003$.

Finally, we explored the power of cosmic chronometers in breaking degeneracies between parameters, and hence to constrain quantities that do not directly affects the expansion history of the Universe, namely the number of relativistic species and the total mass of neutrinos. Our measurements provide a value $N_{\text {eff }}=3.17 \pm 0.15$ that excludes at more than $5 \sigma$ the presence of an extra sterile neutrino, putting also a limit on the sum of neutrino masses, $\Sigma m_{\nu}<0.27 \mathrm{eV}$ at $95 \%$ confidence level.

It is interesting to note that, even if below two sigma, the $w(z)$ obtained from the Chebyshev expansion appears to slightly deviate from a cosmological constant at high redshift. This deviation is, however, not significant with the current data; future surveys, such as Euclid [44], WFIRST [45], DESI [46] and LSST [47], will therefore represent the next significant step, providing sub-percent measurements of the dark energy EoS, and giving fundamental insights for our comprehension of the elusive nature of dark energy.

\section{Acknowledgments}

MM, AC and LP acknowledge financial contributions by grants ASI/INAF I/023/12/0, PRIN MIUR 2010-2011 "The dark Universe and the cosmic evolution of baryons: from current surveys to Euclid", and PRIN MIUR 2015 "Cosmology and Fundamental Physics: illuminating the Dark Universe with Euclid". LV and RJ acknowledge support by AYA2014-58747-P and MDM-2014- 0369 of ICCUB (Unidad de Excelencia Maria de Maeztu). We would like 

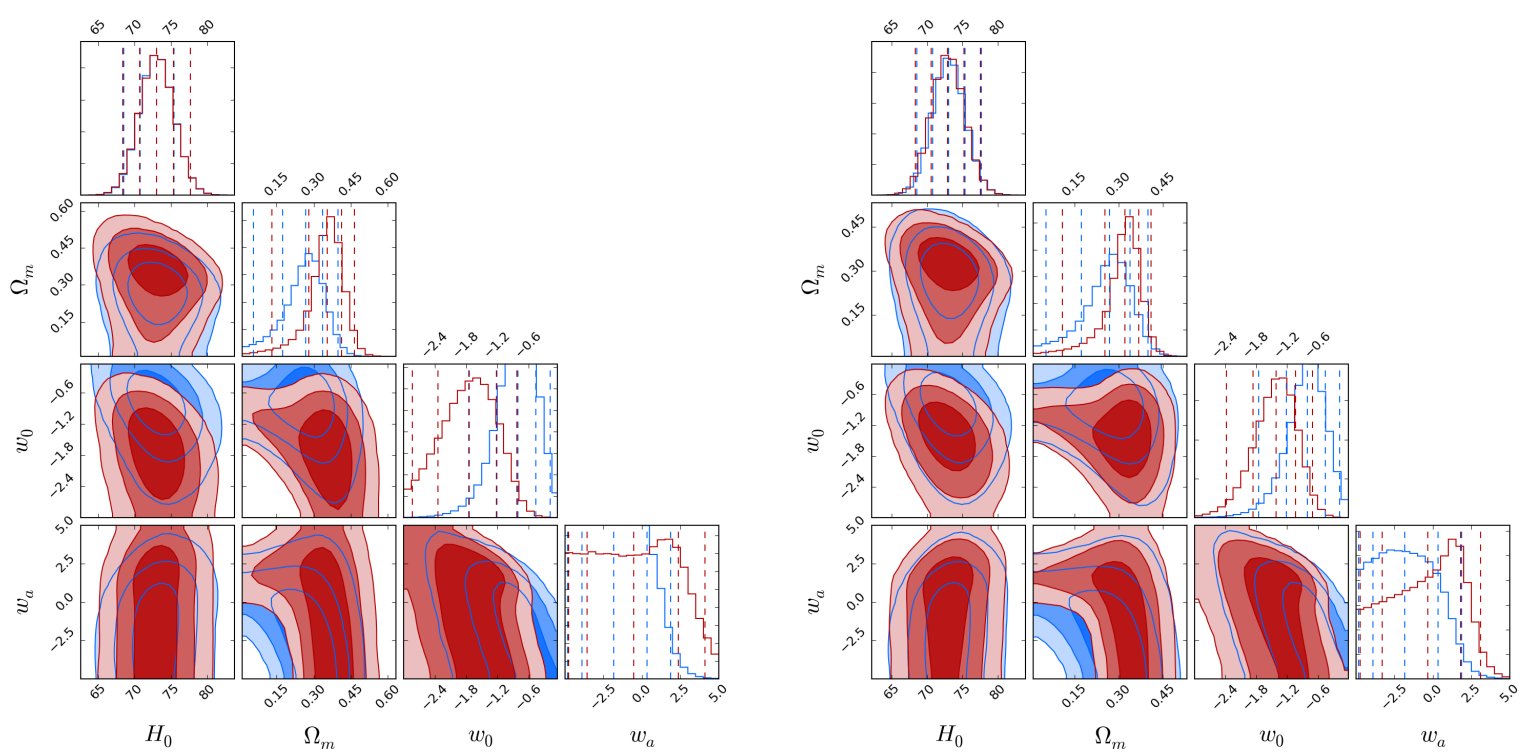

Figure 10. Comparison of the results obtained fitting CC with BC03 (in red) and M11 models (in blue). In the left panel are shown the constraints using measurements taken only from refs. [2, 3, 6], while in the right panel are also considered additional measurements obtained with BC03 model from refs. [11-13]. For this constraints, we assumed a Gaussian prior on $H_{0}=73 \pm 2.4 \mathrm{~km} / \mathrm{s} / \mathrm{Mpc}$ from refs. $[30,31,39]$.

to thank the anonymous referee for the useful comments and suggestions, which helped to improve the paper.

\section{A Dependence of the results on the assumed evolutionary stellar popula- tion synthesis model}

To measure the Hubble parameter $H(z)$ within the CC approach, an evolutionary stellar population synthesis (EPS) model has to be assumed, to calibrate the relation between age and age-related observables used in our analysis. Amongst the models available in literature, so far BC03 [18] and M11 [19] have been used, encompassing significant differences in stellar phases considered, methods implemented and models adopted; for a more detailed discussion, we refer to ref. [3]. In the following, we explore the dependence of our results on the assumed EPS model.

As a reference, we consider the f $w_{0} w_{a} \mathrm{CDM}$ model, and fitted CC data obtained assuming BC03 and M11 models. We performed two different test: firstly, we consider only the limited dataset for which both BC03 and M11 measurements are available [2, 3, 6], and then we compare the result obtained for the full BC03 sample, i.e. by considering also the measurements from refs. [11-13] (for these measurements, only BC03 constraints are available). We also explored how the constraints change by adding the local measurements of $H_{0}$, as a pivot value for the $H(z)$ relation.

The results are shown in figure 10, and reported in table 6 . We find no significant difference between the two constraints, with only M11 measurements pointing to a value of $H_{0}$ at higher odds than $\mathrm{BC} 03$ with respect to state-of-art measurement. The measurements are still well compatible also when a Gaussian prior on $H_{0}$ is assumed, with the full 


\begin{tabular}{|ccccc|}
\hline \multicolumn{5}{|c|}{ MARGINALIZED 1D CONSTRAINTS } \\
f $w_{0} w_{a}$ CDM model \\
\hline & $\mathrm{H}_{0}$ & $\Omega_{\mathrm{m}}$ & $w_{0}$ & $w_{a}$ \\
\hline $\mathrm{BC} 03[2,3,6]$ & $74.2_{-15.8}^{+20.1}$ & $0.33_{-0.19}^{+0.26}$ & $-1.86_{-1.1}^{+1.61}$ & $-0.8_{-4}^{+5.2}$ \\
$\mathrm{M} 11[2,3,6]$ & $78.3_{-15.2}^{+19.6}$ & $0.23_{-0.18}^{+0.18}$ & $-1.19_{-1.5}^{+1.1}$ & $-1.1_{-3.7}^{+4.7}$ \\
$\mathrm{BC} 03[2,3,6,11-13]$ & $73.4_{-13.4}^{+19.2}$ & $0.3_{-0.2}^{+0.18}$ & $-1.46_{-1.4}^{+1.27}$ & $-0.5_{-4.2}^{+4.5}$ \\
\hline $\mathrm{BC} 03[2,3,6]+\mathrm{H}_{0}$ & - & $0.36_{-0.23}^{+0.11}$ & $-1.73_{-1.1}^{+0.9}$ & $-0.5_{-4.2}^{+4.6}$ \\
$\mathrm{M} 11[2,3,6]+\mathrm{H}_{0}$ & - & $0.27_{-0.21}^{+0.13}$ & $-0.81_{-0.9}^{+0.6}$ & $-1.8_{-3}^{+3.7}$ \\
$\mathrm{BC} 03[2,3,6,11-13]+\mathrm{H}_{0}$ & - & $0.32_{-0.21}^{+0.09}$ & $-1.4_{-1}^{+0.7}$ & $-0.3_{-4.4}^{+3.4}$ \\
\hline
\end{tabular}

Table 6. Comparison of the constraints obtained by fitting CC assuming different EPS (BC03 and M11) on $\mathrm{H}_{0}, \Omega_{\mathrm{m}}, w_{0}$ and $w_{a}$ at $95 \%$ confidence level obtained for a flat $\Lambda$ CDM cosmology with a CPL parameterisation for the dark energy EoS. In the lower part of the table, we assumed a Gaussian prior on $H_{0}=73 \pm 2.4 \mathrm{~km} / \mathrm{s} / \mathrm{Mpc}$ from refs. [30,31,39], and hence do not provide the constraints for $H_{0}$.

BC03 dataset providing tighter constraints on cosmological parameters, as expected given the higher number of data available.

We therefore conclude that our measurements are robust, and do not significantly depend on the assumed EPS model.

\section{References}

[1] R. Jimenez and A. Loeb, Constraining cosmological parameters based on relative galaxy ages, Astrophys. J. $\mathbf{5 7 3}$ (2002) 37 [astro-ph/0106145] [INSPIRE].

[2] M. Moresco et al., Improved constraints on the expansion rate of the Universe up to $z \sim 1.1$ from the spectroscopic evolution of cosmic chronometers, JCAP 08 (2012) 006 [arXiv: 1201.3609] [INSPIRE].

[3] M. Moresco et al., A 6\% measurement of the Hubble parameter at $z \sim 0.45$ : direct evidence of the epoch of cosmic re-acceleration, JCAP 05 (2016) 014 [arXiv:1601.01701] [INSPIRE].

[4] M. Moresco, R. Jimenez, A. Cimatti and L. Pozzetti, Constraining the expansion rate of the Universe using low-redshift ellipticals as cosmic chronometers, JCAP 03 (2011) 045 [arXiv: 1010.0831] [INSPIRE].

[5] M. Moresco, L. Verde, L. Pozzetti, R. Jimenez and A. Cimatti, New constraints on cosmological parameters and neutrino properties using the expansion rate of the Universe to $z \sim 1.75$, JCAP 07 (2012) 053 [arXiv: 1201.6658] [INSPIRE].

[6] M. Moresco, Raising the bar: new constraints on the Hubble parameter with cosmic chronometers at $z \sim 2$, Mon. Not. Roy. Astron. Soc. 450 (2015) L16 [arXiv:1503.01116] [INSPIRE].

[7] J. Pforr, C. Maraston and C. Tonini, Recovering galaxy stellar population properties from broad-band spectral energy distribution fitting, Mon. Not. Roy. Astron. Soc. 422 (2012) 3285 [arXiv:1203.3548] [INSPIRE]. 
[8] D.M. Wilkinson et al., P-MaNGA: Full spectral fitting and stellar population maps from prototype observations, Mon. Not. Roy. Astron. Soc. 449 (2015) 328 [arXiv:1503.01124] [INSPIRE].

[9] C. Maraston, Evolutionary population synthesis: Models, analysis of the ingredients and application to high-z galaxies, Mon. Not. Roy. Astron. Soc. 362 (2005) 799 [astro-ph/0410207] [INSPIRE].

[10] Planck collaboration, P.A.R. Ade et al., Planck 2015 results. XIII. Cosmological parameters, Astron. Astrophys. 594 (2016) A13 [arXiv:1502.01589] [InSPIRE].

[11] J. Simon, L. Verde and R. Jimenez, Constraints on the redshift dependence of the dark energy potential, Phys. Rev. D 71 (2005) 123001 [astro-ph/0412269] [INSPIRE].

[12] D. Stern, R. Jimenez, L. Verde, M. Kamionkowski and S.A. Stanford, Cosmic Chronometers: Constraining the Equation of State of Dark Energy. I: H(z) Measurements, JCAP 02 (2010) 008 [arXiv:0907.3149] [INSPIRE].

[13] C. Zhang, H. Zhang, S. Yuan, S. Liu, T.-J. Zhang and Y.-C. Sun, Four new observational H(z) data from luminous red galaxies in the Sloan Digital Sky Survey data release seven, Res. Astron. Astrophys. 14 (2014) 1221 [arXiv: 1207.4541] [InSPIRE].

[14] SDSS collaboration, M. Betoule et al., Improved cosmological constraints from a joint analysis of the SDSS-II and SNLS supernova samples, Astron. Astrophys. 568 (2014) A22 [arXiv: 1401.4064] [INSPIRE].

[15] F. Beutler et al., The 6dF Galaxy Survey: Baryon Acoustic Oscillations and the Local Hubble Constant, Mon. Not. Roy. Astron. Soc. 416 (2011) 3017 [arXiv:1106.3366] [InSPIRE].

[16] A.J. Ross, L. Samushia, C. Howlett, W.J. Percival, A. Burden and M. Manera, The clustering of the SDSS DR7 main Galaxy sample - I. A 4 per cent distance measure at $z=0.15$, Mon. Not. Roy. Astron. Soc. 449 (2015) 835 [arXiv:1409.3242] [InSPIRE].

[17] BOSS collaboration, L. Anderson et al., The clustering of galaxies in the SDSS-III Baryon Oscillation Spectroscopic Survey: baryon acoustic oscillations in the Data Releases 10 and 11 Galaxy samples, Mon. Not. Roy. Astron. Soc. 441 (2014) 24 [arXiv:1312.4877] [InSPIRE].

[18] G. Bruzual and S. Charlot, Stellar population synthesis at the resolution of 2003, Mon. Not. Roy. Astron. Soc. 344 (2003) 1000 [astro-ph/0309134] [INSPIRE].

[19] C. Maraston and G. Strömbäck, Stellar population models at high spectral resolution, Mon. Not. Roy. Astron. Soc. 418 (2011) 2785 [arXiv:1109.0543] [InSPIRE].

[20] SNLS collaboration, P. Astier et al., The Supernova legacy survey: Measurement of $\Omega_{M}, \Omega_{\Lambda}$ and $w$ from the first year data set, Astron. Astrophys. 447 (2006) 31 [astro-ph/0510447] [INSPIRE].

[21] SNLS collaboration, M. Sullivan et al., SNLS3: Constraints on Dark Energy Combining the Supernova Legacy Survey Three Year Data with Other Probes, Astrophys. J. 737 (2011) 102 [arXiv: 1104.1444] [INSPIRE].

[22] A.G. Riess et al., New Hubble Space Telescope Discoveries of Type Ia Supernovae at $z \geq 1$ : Narrowing Constraints on the Early Behavior of Dark Energy, Astrophys. J. 659 (2007) 98 [astro-ph/0611572] [INSPIRE].

[23] N. Suzuki et al., The Hubble Space Telescope Cluster Supernova Survey: V. Improving the Dark Energy Constraints Above $z>1$ and Building an Early-Type-Hosted Supernova Sample, Astrophys. J. 746 (2012) 85 [arXiv:1105.3470] [INSPIRE].

[24] SNLS collaboration, A. Conley et al., Supernova Constraints and Systematic Uncertainties from the First 3 Years of the Supernova Legacy Survey, Astrophys. J. Suppl. 192 (2011) 1 [arXiv: 1104.1443] [INSPIRE]. 
[25] A.J. Cuesta, L. Verde, A. Riess and R. Jimenez, Calibrating the cosmic distance scale ladder: the role of the sound horizon scale and the local expansion rate as distance anchors, Mon. Not. Roy. Astron. Soc. 448 (2015) 3463 [arXiv:1411.1094] [INSPIRE].

[26] É. Aubourg et al., Cosmological implications of baryon acoustic oscillation measurements, Phys. Rev. D 92 (2015) 123516 [arXiv:1411.1074] [INSPIRE].

[27] E.A. Kazin et al., The WiggleZ Dark Energy Survey: improved distance measurements to $z=1$ with reconstruction of the baryonic acoustic feature, Mon. Not. Roy. Astron. Soc. 441 (2014) 3524 [arXiv: 1401.0358 ] [INSPIRE].

[28] A. Veropalumbo, F. Marulli, L. Moscardini, M. Moresco and A. Cimatti, An improved measurement of baryon acoustic oscillations from the correlation function of galaxy clusters at $z \sim 0.3$, Mon. Not. Roy. Astron. Soc. 442 (2014) 3275 [arXiv:1311.5895] [InSPIRE].

[29] A. Veropalumbo, F. Marulli, L. Moscardini, M. Moresco and A. Cimatti, Measuring the distance-redshift relation with the baryon acoustic oscillations of galaxy clusters, Mon. Not. Roy. Astron. Soc. 458 (2016) 1909 [arXiv: 1510.08852] [INSPIRE].

[30] A.G. Riess et al., A 3\% Solution: Determination of the Hubble Constant with the Hubble Space Telescope and Wide Field Camera 3, Astrophys. J. 730 (2011) 119 [Erratum ibid. 732 (2011) 129] [arXiv: 1103.2976] [INSPIRE].

[31] E.M.L. Humphreys, M.J. Reid, J.M. Moran, L.J. Greenhill and A.L. Argon, Toward a New Geometric Distance to the Active Galaxy NGC 4258. III. Final Results and the Hubble Constant, Astrophys. J. 775 (2013) 13 [arXiv:1307.6031] [INSPIRE].

[32] G. Efstathiou, $H_{0}$ Revisited, Mon. Not. Roy. Astron. Soc. 440 (2014) 1138 [arXiv:1311.3461] [INSPIRE].

[33] L. Verde, P. Protopapas and R. Jimenez, Planck and the local Universe: Quantifying the tension, Phys. Dark Univ. 2 (2013) 166 [arXiv:1306.6766] [INSPIRE].

[34] Planck collaboration, P.A.R. Ade et al., Planck 2013 results. XVI. Cosmological parameters, Astron. Astrophys. 571 (2014) A16 [arXiv:1303.5076] [InSPIRE].

[35] M. Chevallier and D. Polarski, Accelerating universes with scaling dark matter, Int. J. Mod. Phys. D 10 (2001) 213 [gr-qc/0009008] [InSPIRE].

[36] E.V. Linder, Exploring the expansion history of the universe, Phys. Rev. Lett. 90 (2003) 091301 [astro-ph/0208512] [INSPIRE].

[37] D. Foreman-Mackey, D.W. Hogg, D. Lang and J. Goodman, emcee: The MCMC Hammer, Publ. Astron. Soc. Pac. 125 (2013) 306 [arXiv:1202.3665] [InSPIRE].

[38] J. Goodman and J. Weare, Ensamble samplers with affine invariance, Commun. Appl. Math. Comput. Sci. 5 (2010) 65.

[39] A.J. Cuesta et al., The clustering of galaxies in the SDSS-III Baryon Oscillation Spectroscopic Survey: Baryon Acoustic Oscillations in the correlation function of LOWZ and CMASS galaxies in Data Release 12, Mon. Not. Roy. Astron. Soc. 457 (2016) 1770 [arXiv: 1509.06371] [INSPIRE].

[40] B.A. Reid, L. Verde, R. Jimenez and O. Mena, Robust Neutrino Constraints by Combining Low Redshift Observations with the CMB, JCAP 01 (2010) 003 [arXiv:0910.0008] [INSPIRE].

[41] F. de Bernardis, A. Melchiorri, L. Verde and R. Jimenez, The Cosmic Neutrino Background and the Age of the Universe, JCAP 03 (2008) 020 [arXiv:0707.4170] [INSPIRE].

[42] V. Barger, E. Guarnaccia and D. Marfatia, Classification of dark energy models in the $\left(w_{0}, w_{a}\right)$ plane, Phys. Lett. B 635 (2006) 61 [hep-ph/0512320] [INSPIRE].

[43] R.R. Caldwell and E.V. Linder, The Limits of quintessence, Phys. Rev. Lett. 95 (2005) 141301 [astro-ph/0505494] [INSPIRE]. 
[44] EUCLID collaboration, R. Laureijs et al., Euclid Definition Study Report, arXiv:1110.3193 [INSPIRE].

[45] D. Spergel et al., Wide-Field InfraRed Survey Telescope-Astrophysics Focused Telescope Assets WFIRST-AFTA Final Report, arXiv: 1305.5422 [INSPIRE].

[46] DESI collaboration, M. Levi et al., The DESI Experiment, a whitepaper for Snowmass 2013, arXiv: 1308.0847 [INSPIRE].

[47] LSST Science, LSST Project collaboration, P.A. Abell et al., LSST Science Book, Version 2.0, arXiv: 0912.0201 [INSPIRE]. 